\title{
Clinical significance and biological role of Wnt10a in ovarian cancer
}

\author{
PING LI, WENLIAN LIU, QIAN XU and CHANGXIU WANG \\ Department of Obstetrics and Gynecology, Linyi People's Hospital, Linyi, Shandong 276000, P.R. China
}

Received December 15, 2015; Accepted May 25, 2017

DOI: $10.3892 / \mathrm{ol} .2017 .7062$

\begin{abstract}
Ovarian cancer is one of the five most malignant types of cancer in females, and the only currently effective therapy is surgical resection combined with chemotherapy. Wnt family member 10A (Wnt10a) has previously been identified to serve an oncogenic function in several tumor types, and was revealed to have clinical significance in renal cell carcinoma; however, there is still only limited information regarding the function of Wnt10a in the carcinogenesis of ovarian cancer. The present study identified increased expression levels of Wnt10a in two cell lines, SKOV3 and A2780, using reverse transcription-polymerase chain reaction. Functional analysis indicated that the viability rate and migratory ability of SKOV3 cells was significantly inhibited following Wnt10a knockdown using short interfering RNA (siRNA) technology. The viability rate of SKOV3 cells decreased by $\sim 60 \%$ compared with the control and the migratory ability was only $\sim 30 \%$ of that in the control. Furthermore, the expression levels of $\beta$-catenin, transcription factor 4, lymphoid enhancer binding factor 1 and cyclin D1 were significantly downregulated in SKOV3 cells treated with Wnt10a-siRNA3 or LGK-974, a specific inhibitor of the canonical Wnt signaling pathway. However, there were no synergistic effects observed between Wnt10a siRNA3 and LGK-974, which indicated that Wnt10a activated the Wnt/ $\beta$-catenin signaling pathway in SKOV3 cells. In addition, using quantitative PCR, Wnt10a was overexpressed in the tumor tissue samples obtained from 86 patients with ovarian cancer when compared with matching paratumoral tissues. Clinicopathological association analysis revealed that Wnt10a was significantly associated with high-grade (grade III, $\mathrm{P}=0.031)$ and late-stage $(\mathrm{T} 4, \mathrm{P}=0.008)$ ovarian cancer. Furthermore, the estimated 5-year survival rate was $18.4 \%$ for patients with low Wnt10a expression levels $(n=38)$, whereas for patients with high Wnt10a expression $(n=48)$ the
\end{abstract}

Correspondence to: Dr Wenlian Liu, Department of Obstetrics and Gynecology, Linyi People's Hospital, 27 Liberate Road, Linyi, Shandong 276000, P.R. China

E-mail: liuwl_0001@163.com

Key words: Wnt10a, Wnt//-catenin signaling, ovarian cancer, viability, migration rate was $6.3 \%$. The results of the present study suggested that Wnt10a serves an oncogenic role during the carcinogenesis and progression of ovarian cancer via the Wnt/ $\beta$-catenin signaling pathway.

\section{Introduction}

Ovarian cancer is the most serious cause of gynecologic cancer morbidity and ranks as the fifth highest cause of cancer-associated mortality in females (1). It was estimated that $\sim 21,290$ novel cases of ovarian cancer and $\sim 14,180$ ovarian cancer-associated mortalities may occur in the US in $2015(1,2)$. The majority of patients with ovarian cancer were diagnosed with stage III or IV, and $<30 \%$ of patients with advanced ovarian cancer survive $>5$ years following the initial diagnosis (2). Furthermore, $75-80 \%$ of females with advanced ovarian cancer may experience tumor progression or recurrence following initial therapy (2). The primary type of therapy available for ovarian cancer has remained surgical resection accompanied by chemotherapy with platinum, taxane or other chemotherapeutic agents; however, traditional therapy is typically ineffective if tumor metastasis or relapse has occurred $(2,3)$. Therefore, the underlying molecular mechanism of ovarian cancer and the development of novel targeted drugs, as well as a more effective strategy for patients with ovarian cancer, requires investigation.

The Wnt/ $\beta$-catenin signaling pathway serves vital functions during embryonic development and the maintenance of homeostasis (4-6). The 19 members of the Wnt family are categorized into two classes, on the basis of $\beta$-catenin involvement: The canonical pathway $(\beta$-catenin-dependent) and the non-canonical pathway ( $\beta$-catenin-independent). In the canonical pathway, Wnt ligands bind to the frizzled receptor and activate Disheveled, inhibiting the phosphorylation of $\beta$-catenin by glycogen synthase kinase $3-\beta$ and resulting in the accumulation of $\beta$-catenin (4-6). Subsequently, $\beta$-catenin undergoes nuclear translocation from the cytoplasm and activates certain downstream target genes, including cyclin D1, transcription factor 4 (TCF4), and lymphoid enhancer binding factor 1 (LEF1) (2,7).

The 19 members of the Wnt family have been identified in a number of diseases and tumor types, including ovarian cancer, and have typically been identified to promote cancer initiation or progression by regulating cell viability, migration and apoptosis (8-11). In the majority of tumor types, the 
mutation of key components in the Wnt signaling pathway, including $\beta$-catenin, Axin-1 and adenomatous polyposis coli, was common (8). However, in ovarian cancer (including clear cell, epithelial and mucinous ovarian cancer), with the exception of endometrioid ovarian cancer, other underlying molecular mechanisms may serve dominant functions $(8,10)$. For example, Wnt7a accelerated tumor viability and progression in advanced-stage ovarian cancer by activating the Wnt/ $\beta$-catenin signaling pathway (12). Wnt10b was identified to be expressed at significantly increased levels in endometrial cancer tissues, compared with those in healthy tissues (13). Wnt10a, homologous with Wnt10b, was also identified to be associated with tooth agenesis (14); however, it was demonstrated that Wnt10a was overexpressed in a number of tumor types, including gastric, colorectal and esophageal cancer, and promyelocytic leukemia (1,15-19). Furthermore, Wnt10a was revealed to promote invasion in esophageal squamous cell carcinoma and function as an oncogene in renal cell carcinoma, by activating the $\mathrm{Wnt} / \beta$-catenin signaling pathway $(16,18)$. However, information regarding the function of Wnt10a in ovarian cancer, aside from the upregulated expression of Wnt10a in endometrial cancer, remains limited.

In the present study, the function of Wnt10a in ovarian cancer was investigated, and it was identified that Wnt10a may improve the viability and migration of the SKOV-3 cell line through the activation of $\mathrm{Wnt} / \beta$-catenin signaling. In addition, Wnt10a was overexpressed in ovarian cancer tissues and associated with high tumor grade and late-stage ovarian cancer. The overall survival time of patients with increased Wnt10a expression was decreased compared with that of patients with normal Wnt10a expression. The results of the present study indicated that Wnt10a serves critical functions in the carcinogenesis and progression of epithelial ovarian cancer via the Wnt/ $\beta$-catenin signaling pathway, which may present a novel therapeutic strategy for female patients with ovarian cancer.

\section{Materials and methods}

Cell lines and ovarian cancer tissues. The A2780 and SKOV3 human ovarian cancer cell lines were purchased from the Cell Bank Type Culture Collection of Chinese Academy of Sciences (Shanghai, China) and maintained in Dulbecco's modified Eagle's medium (DMEM; Gibco; Thermo Fisher Scientific, Inc., Waltham, MA, USA) supplemented with $10 \%$ fetal bovine serum (FBS; Gibco; Thermo Fisher Scientific, Inc.), $100 \mathrm{U} / \mathrm{ml}$ penicillin and $100 \mu \mathrm{g} / \mathrm{ml}$ streptomycin, at $37^{\circ} \mathrm{C}$ in humidified incubator containing $5 \% \mathrm{CO}_{2}$.

Frozen resected surgical tissues from a total of 86 patients (age range, 32-67 years; mean age, 52.4 years) with ovarian cancer between July 2006 and December 2010 were collected from the Department of Obstetrics and Gynecology of Linyi People's Hospital (Linyi, China). All tumor tissues were diagnosed by a pathologist prior to use in the present study according to the World Health Organization criteria and the Federation International of Gynecology and Obstetrics (FIGO) guidelines (20-22). A total of 30 patients with early tumor stage (T1/T2) were selected and underwent primary cytoreductive surgery, the other 56 patients with late tumor stage (T3/T4) received postoperative chemotherapy following bilateral oophorectomy. All patients used for further study had a complete clinicopathological information including 5-year follow-up (three times a year during the first two years and twice a year during the later years). The development and pathogenic progression of each patient with ovarian cancer was diagnosed and classified by histological examination, according to the World Health Organization criteria (20). Written informed consent was obtained from individual subjects and the experimental protocols were approved by the Ethics Committee of Linyi People's Hospital (Linyi, China). All experiments complied with the current laws in China.

Reverse transcription-polymerase chain reaction (RT-PCR) and RT-quantitative PCR (RT-qPCR). Total RNA from ovarian cancer tissues or ovarian cancer cells was extracted using TRIzol ${ }^{\circledR}$ (Invitrogen; Thermo Fisher Scientific, Inc.), followed by treatment with RQ1RNase-free DNase (Promega Corporation, Madison, WI, USA). cDNA was prepared from $2 \mu \mathrm{g}$ total RNA using SuperScript ${ }^{\mathrm{TM}}$ III (Invitrogen; Thermo Fisher Scientific, Inc.), according to the manufacturer's protocol. A total of $2 \mu \mathrm{l}$ synthesized cDNA was used as template for RT-PCR in a $20 \mu 1$ reaction mixture, containing 10 pmol of each primer (listed in Table I), 2 U recombinant Taq DNA polymerase (Invitrogen; Thermo Fisher Scientific, Inc.), $2.0 \mu 1$ $1 \mathrm{X}$ reaction buffer and 200 pmol dNTPs. The protocol was as follows, for 35 cycles: Denaturation, $95^{\circ} \mathrm{C}$ for $30 \mathrm{sec}$; annealing, $58^{\circ} \mathrm{C}$ for $30 \mathrm{sec}$; extension, $72^{\circ} \mathrm{C}$ for $1 \mathrm{~min}$. Subsequently, the products were incubated at $72^{\circ} \mathrm{C}$ for $10 \mathrm{~min}$. The PCR products were run on agarose ( $2 \%$ gel) and visualized by staining with ethidium bromide. RT-qPCR was conducted using the standard SYBR-Green PCR kit (Takara Bio, Inc., Otsu, Japan) to evaluate the relative mRNA levels of Wnt10a and GAPDH (as the internal control) in the aforementioned ovarian cancer cell lines and ovarian tumor tissues on the ABI 7500 real-time PCR system (Applied Biosystems, Foster City, CA, USA). The protocol was as follows: $94^{\circ} \mathrm{C}$ for 1 min followed by 35 cycles of $94^{\circ} \mathrm{C}$ for $1 \mathrm{~min}$ and $53^{\circ} \mathrm{C}$ for $2 \mathrm{~min}$. Data were analyzed using ABI 7500 software (version 2.0.6; Applied Biosystems) and presented in terms of relative quantification (RQ) to GAPDH, on the basis of $2^{-\Delta \mathrm{Cq}}$, where $\Delta \mathrm{Cq}=\mathrm{Cq}$ (target) - $\mathrm{Cq}$ (reference). Fold change was calculated using the $2^{-\Delta \Delta C q}$ method (23). All samples were examined in triplicate. Primers used for RT-PCR and RT-qPCR are listed in Table I.

Transfection of SKOV3 cells with Wnt10a short interfering (si)RNA. The Wnt10a-siRNA and the negative control siRNA, summarized in Table I, were designed and synthesized by Shanghai Gene Pharma (Shanghai, China). SKOV3 cells were transiently transfected with Wnt10a-siRNA fragments using Lipofectamine ${ }^{\circledR} 2000$ (Invitrogen; Thermo Fisher Scientific, Inc.) according to the manufacturer's protocol. SKOV3 cells in the exponential growth phase were seeded $\left(1 \times 10^{4}\right.$ cells $\left./ \mathrm{ml}\right)$ into 12 -well plates. After $24 \mathrm{~h}$, cells were transfected with 100 pmol Wnt10a-siRNA using Lipofectamine 2000. DMEM medium was replaced with $1 \mathrm{ml}$ DMEM, containing $10 \% \mathrm{FBS}$, after $12 \mathrm{~h}$ of incubation at $37^{\circ} \mathrm{C}$ and subsequently transfected cells were cultured at $37^{\circ} \mathrm{C}$ in an atmosphere containing $5 \%$ $\mathrm{CO}_{2}$, prior to further treatment.

MTT assay. Cell viability was evaluated using the MTT assay. SKOV3 cells, transfected with Wnt10a siRNA, were seeded 
Table I. Sequences of primers for RT-PCR and RT-qPCR, and the siRNA targets.

\begin{tabular}{llll}
\hline Experiment & Gene & Direction/number & Sequence, 5'-3' $^{\prime}$ \\
\hline qPCR primers & Wnt10a & Forward & TCGCAACAAGATCCCCTATGA \\
& GAPDH & Reverse & CAGGGCACACGCATTGGACA \\
& & Forward & TGGAGCCAAAAGGGTCATCA \\
RT-PCR primers & Reverse & TCGCAACAAGATCCCCTATG \\
& Wnt10a & Forward & GCAGTGCATCCAGTTGTAAG \\
GAPDH & Reverse & AGGAGCGAGATCCCTCCA \\
siRNA target & Forward & CCGTTCAGCTCAGGGATGAC \\
& & Reverse & GGTCAGCACCCAATGACAT \\
& & siRNA1 & GCCAACACAGTGTGCCTAA \\
& & siRnA2 & GCGAGAATGAGGCTTCACA
\end{tabular}

RT-PCR, reverse transcription polymerase chain reaction; qPCR, quantitative PCR; siRNA, short interfering RNA.

into 96-well plates (2x10 cells/well) in $100 \mu 1$ DMEM (Gibco; Thermo Fisher Scientific, Inc.). A total of $20 \mu \mathrm{l}$ MTT solution ( $5 \mathrm{mg} / \mathrm{ml}$; Sigma-Aldrich; Merck KGaA, Darmstadt, Germany) was added into each well daily (on days 2, 3, 4 and 5), and the plates were incubated for $4 \mathrm{~h}$ at $37^{\circ} \mathrm{C}$ prior to the removal of the supernatant. Subsequently, $100 \mu 1$ dimethyl sulfoxide (Sigma-Aldrich; Merck KGaA) was added to dissolve the crystals. Absorbance was determined at a wavelength of $490 \mathrm{~nm}$ with a microplate reader. Each experiment was performed in triplicate.

Cell migration assay. Following Wnt10a siRNA transfection, a scratch was created, using a p200 pipette tip, and the cells were gently washed twice with PBS to remove the suspended cells. Subsequently, the monolayer cells were cultured in serum-free DMEM for $24 \mathrm{~h}$ at $37^{\circ} \mathrm{C}$. At the designated time point 2 and $24 \mathrm{~h}$ after a scratch was created, the width of each scratch at five randomly selected fields along the scratch were observed under a light microscope (x20 magnification) and recorded. The migration rate was calculated using the following formula: Migration rate $(\%)=\left(\mathrm{S}_{2 \mathrm{~h}}-\mathrm{S}_{24 \mathrm{~h}}\right) / \mathrm{S}_{2 \mathrm{~h}} \times 100 . \mathrm{S}_{2 \mathrm{~h}}$ represents the distance of the scratch at $2 \mathrm{~h}$ and $\mathrm{S}_{24 \mathrm{~h}}$ represents the distance at $24 \mathrm{~h}$.

Western blot analysis. Cells were cultured in 6-well plates at $2 \times 10^{6} /$ well and transfected with Wnt10a-siRNA fragments or treated with $0.4 \mathrm{nM}$ LGK-974, a specific inhibitor for porcupine homolog (Drosophila; PORCN), in Wnt/ $\beta$-catenin signaling pathway. After $48 \mathrm{~h}$ of incubation at $37^{\circ} \mathrm{C}$, total protein was extracted using iced lysis buffer ( $1 \%$ Triton $\mathrm{X}-100$, 50 mM Tris- $\mathrm{HCl}$, pH 7.4; $150 \mathrm{mM} \mathrm{NaCl} ; 0.1 \%$ SDS; $1 \mathrm{mM}$ phenylmethanesulfonyl fluoride; $1 \mathrm{mM}$ EDTA). Subsequently, the total protein concentration was determined using a bicinchoninic acid assay (BCA Protein Assay kit; Generay Biotech Co., Ltd., Shanghai, China). A total of $10 \mu \mathrm{g}$ protein was separated on an 10\% SDS-PAGE gel, transferred to nitrocellulose membranes followed by blocking with $5 \%$ non-fat milk for $2 \mathrm{~h}$ at room temperature, and then incubated overnight with primary mouse monoclonal antibodies against $\beta$-catenin
(1:250, sc65480; Santa Cruz Biotechnology, Inc., Dallas, TX, USA), cyclin D1 (1:400, sc-70899; Santa Cruz Biotechnology, Inc.), LEF1 (1:800, ab137872), TCF4 (1:500, ab217668) and GAPDH (1:2,000, ab181602) (Abcam, Cambridge, MA, USA) at $4^{\circ} \mathrm{C}$. Membranes were subsequently washed and incubated with a horseradish peroxidase-conjugated secondary antibody $\left(1: 10,000\right.$, ab97051) for $1 \mathrm{~h}$ at $25^{\circ} \mathrm{C}$. Immunoreactivity was determined using an enhanced chemiluminescence kit (Merck $\mathrm{KGaA}$ ).

Statistical analysis. All data are expressed as the mean \pm standard deviation of three independent experiments and were analyzed using a two-tailed paired Student's t-test between two groups with SPSS software (version 16.0; SPSS, Inc., Chicago, IL, USA). Associations between Wnt10a expression and clinicopathological characteristics were analyzed using the $\chi^{2}$ test. Survival curves were plotted using the Kaplan-Meier estimator method and compared using the log-rank test. $\mathrm{P}<0.05$ was considered to indicate a statistically significant difference.

\section{Results}

Knockdown of Wnt10a in the SKOV3 human ovarian cancer cell line suppresses cell viability. Using the semi-quantitative RT-PCR, the expression of Wnt10a was determined in two human ovarian cancer cell lines, SKOV3 and A2780. As presented in Fig. 1A, Wnt10a was expressed at an increased level in the two cell lines. To determine the function of Wnt10a in cell viability, three siRNA fragments targeting the coding sequence of Wnt10a were designed and synthesized. Subsequently, the three Wnt10a-siRNA fragments or negative control (NC) were transfected into cell line SKOV3. Following the transfection of Wnt10a-siRNA3 fragment for $48 \mathrm{~h}$, the mRNA level of Wnt10a was significantly decreased, indicating efficient knockdown of Wnt10a in SKOV3 (Fig. 1A and B). Cell viability of Wnt10a-siRNA3-SKOV3 was determined using the MTT assay for five days. The results of the present study identified that the downregulation of Wnt10a inhibited the viability of SKOV3 cells, and on day 5 the viability rate of 
A

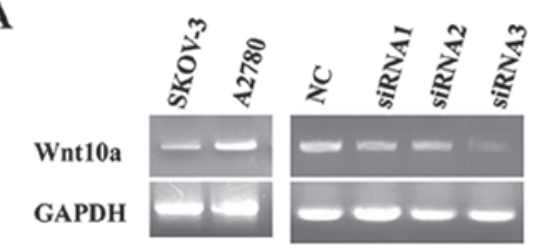

B

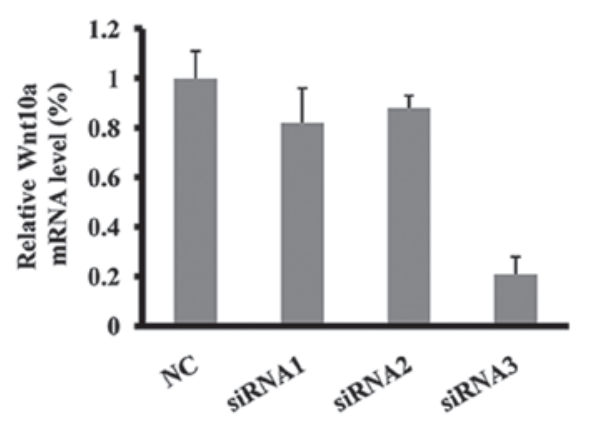

C

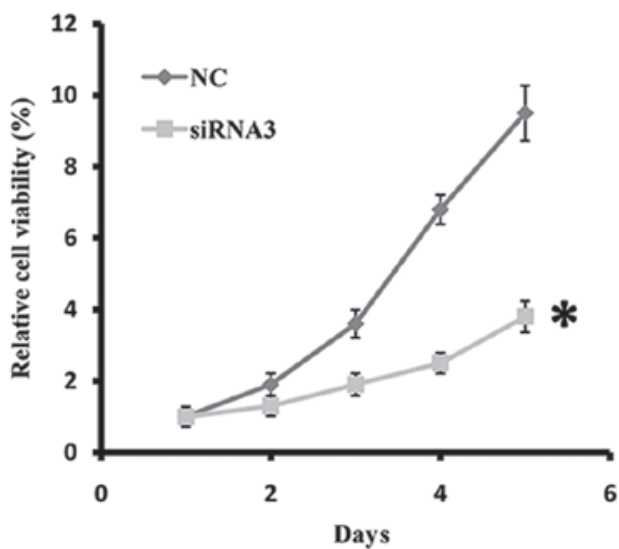

Figure 1. Wnt10a knockdown suppresses the viability of SKOV3 cells. (A) The expression of Wnt10a in two ovarian cancer cell lines (A2780 and SKOV3) and the effect of siRNA on Wnt10a expression, determined using RT-PCR. (B) The knockdown efficiency of Wnt10a in SKOV3 cells, as determined using RT-qPCR. (C) The viability curve of SKOV3 cells transfected with Wnt10a siRNA on five consecutive days. ${ }^{*} \mathrm{P}<0.05$ indicates a significant difference. All data are from experiments conducted in triplicate. RT-PCR, reverse transcription polymerase chain reaction; qPCR, quantitative PCR; NC, negative control; siRNA, short interfering RNA.

$\mathbf{A}$
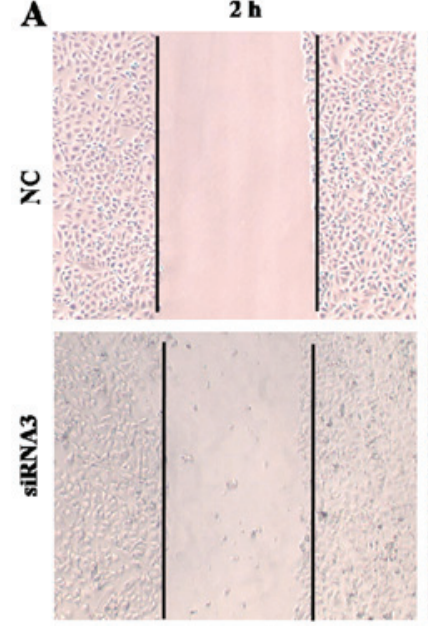

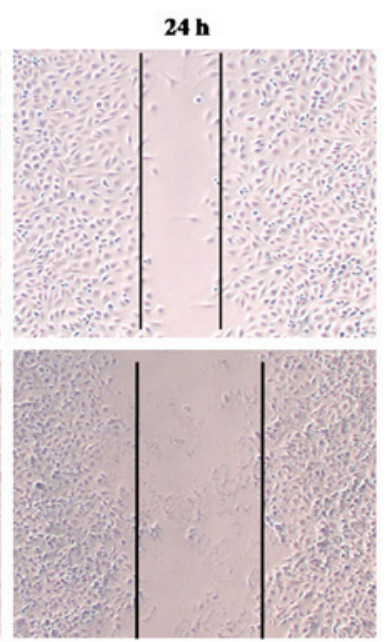

B

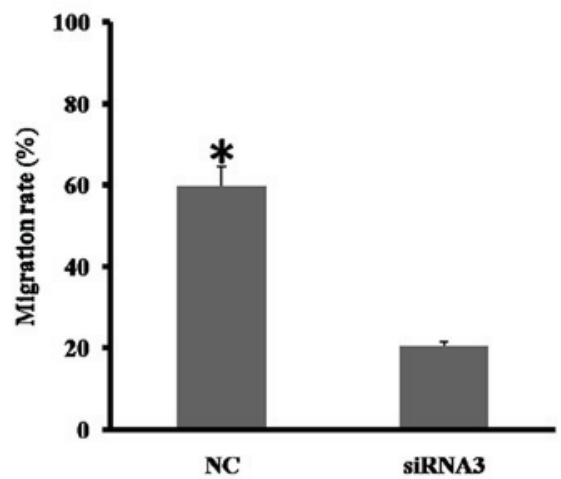

Figure 2. Effects of Wnt10a knockdown on the migratory ability of SKOV3 cells. (A) Wound-healing assay using SKOV3 cells transfected with Wnt10a siRNA3 after $24 \mathrm{~h}$. The migration of cell was observed under a light microscope at x 20 magnification. (B) Migratory rate calculated from the wound-healing assay. "P<0.05 indicates a significant difference. All data were obtained from triplicate experiments. NC, negative control; siRNA, short interfering RNA.

Wnt10a-siRNA3-SKOV3 cells was $40 \%$ that of the control (Fig. 1C). Therefore, knockdown of Wnt10a inhibited the viability of SKOV3 cells.

Knockdown of Wnt10a suppresses the migratory ability of SKOV3 cells. Either migration or invasion is typical for malignant tumor cells, and Wnt10a has been identified to promote the migration of A498 and A786-Orenal cancer cells. The effect of Wnt10a on the motile capability of SKOV3 cells was investigated in the present study using a wound-healing assay. As presented in Fig. 2A, the migratory ability of Wnt10a-siRNA3-SKOV3 cells was significantly decreased, compared with that of the NC group. The migrated distance of Wnt10a-siRNA3-SKOV3 cell was $230 \%$ of that of the NC group (Fig. 2B). The results of the present study demonstrated that decreased Wnt10a expression disrupts the migratory ability of SKOV3 cells.

Wnt10a activates the Wnt/ $\beta$-catenin signaling pathway. A previous study reported that Wnt10a activates the Wnt/ $\beta$-catenin signaling pathway in renal carcinoma cells (18). Therefore, we hypothesized that the knockdown of Wnt10a in SKOV3 cells may disrupt the Wnt/ $\beta$-catenin signaling pathway. As predicted, the expression of molecules in the Wnt/ $\beta$-catenin signaling pathway, including $\beta$-catenin, TCF4, LEF1 and cyclin D1, was significantly downregulated in Wnt10a-siRNA3-SKOV3 cells (Fig. 3A), which was consistent with the effect of LGK-974, a specific inhibitor for porcupine homolog (Drosophila; PORCN), in Wnt//-catenin signaling pathway. In addition, either Wnt10a-siRNA3 or LGK-974 may 
Table II. Association between the expression of Wnt10a and clinicopathological characteristics in ovarian cancer.

\begin{tabular}{lcccc}
\hline & \multicolumn{3}{c}{ Wnt10a expression level } & \\
\cline { 3 - 4 } Characteristic & $\mathrm{n}$ & $\leq 4$-fold & >4-fold & P-value \\
\hline Total & 86 & 37 & 49 & \\
Age, years & & & & 0.829 \\
$\quad 50$ & 48 & 20 & 28 & \\
$>50$ & 38 & 17 & 21 & \\
TNM stage & & & & $0.008^{\mathrm{a}}$ \\
Early (T1/T2) & 30 & 22 & 8 & \\
Late (T3/T4) & 56 & 15 & 41 & \\
Grade & & & & $0.031^{\mathrm{a}}$ \\
Low & 33 & 19 & 14 & \\
High & 53 & 18 & 35 & \\
Histology & & & & \\
Clear cell & 39 & 16 & 23 & \\
Other & 47 & 21 & 26 & \\
\hline
\end{tabular}

${ }^{\text {a }}<<0.05$. TNM, tumor-node-metastasis.

inhibit the viability of SKOV3; however, there was no synergic effect exhibited between siRNA3 and LGK-974 (Fig. 3B), suggesting that $\mathrm{Wnt} / \beta$-catenin signaling is inhibited by Wnt10a siRNA. Therefore, Wnt10a was identified to promote the viability and migration of SKOV3 cells through activation of the Wnt $/ \beta$-catenin signaling pathway.

Wntloa overexpression is associated with carcinogenesis of ovarian cancer and poor survival. The results of the present study identified that Wnt10a may serve an oncogenic role during ovarian cancer carcinogenesis; however, the clinical significance of Wnt10a in ovarian cancer progression remains unknown. To evaluate the clinical significance of Wnt10a, RT-qPCR was employed to determine the level of Wnt10a in tumoral and paratumoral tissues from 86 patients with ovarian cancer. As presented in Fig. 4, overexpression of Wnt10a was significantly associated with high-grade (grade III, $\mathrm{P}=0.031$ ) and late-stage $(\mathrm{T} 3, \mathrm{P}=0.008)$; however, overexpression of Wnt10a was not associated with other clinical characteristics (Table II), including age and histology. Furthermore, the estimated 5-year survival rate was $18.4 \%$ for patients with decreased Wnt10a expression $(n=38)$, whereas it was $6.3 \%$ for patients with increased Wnt10a expression $(n=48)$, and a significant difference was identified between these two groups $(\mathrm{P}=0.019)$. The median survival time was $20 \pm 4.45$ months for patients with low Wnt10a levels, which was elevated when compared with that for patients with high Wnt10a levels (13 \pm 3.67 months).

\section{Discussion}

The morbidity and mortality caused by ovarian cancer is a threat to the health of females. Although efforts have been made to increase the quantity of novel molecule-targeted
A

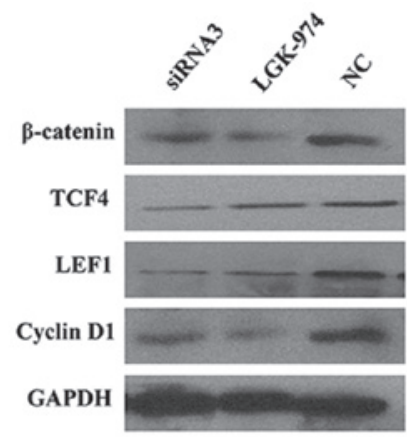

B

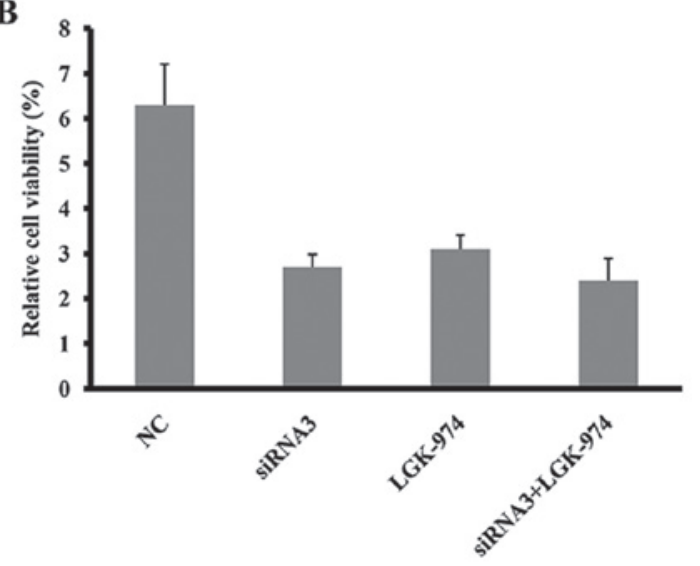

Figure 3. Wnt10a knockdown inactivated the Wnt/ $\beta$-catenin signaling pathway. (A) Wnt10a siRNA3 and the Wnt inhibitor LGK-974 downregulated the expression of $\beta$-catenin, TCF, cyclin D1 and LEF1 in SKOV3 cells. (B) The effect of Wnt10a siRNA3 and LGK-974 on the viability of SKOV3 cells on five consecutive days. All data were obtained from triplicate experiments. siRNA, short interfering RNA; TCF, transcription factor; LEF1, lymphoid enhancer binding factor $1 ; \mathrm{NC}$, negative control.

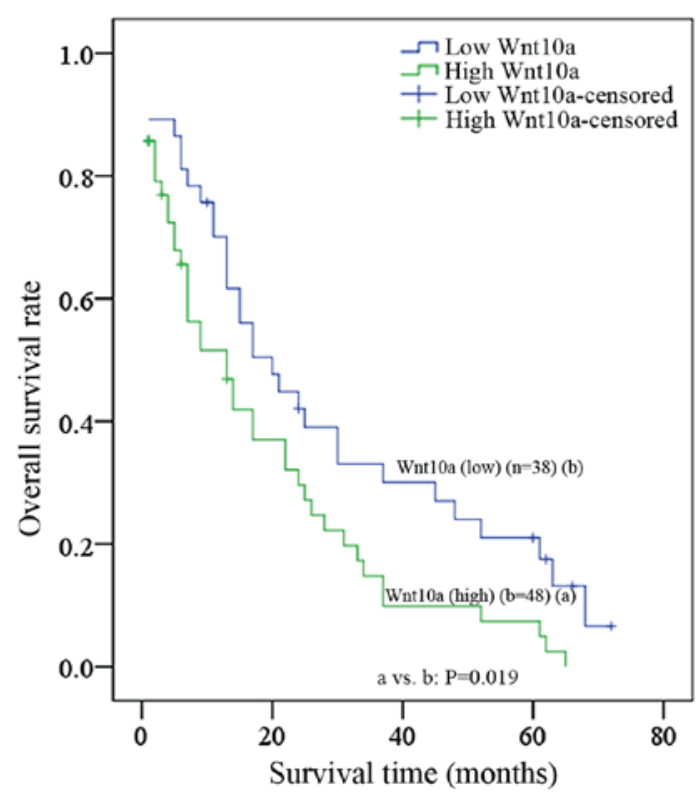

Figure 4. Kaplan-Meier estimator survival curve, according to Wnt10a expression. Survival time of patients with decreased expression of Wnt10a revealed a significantly improved survival time, as compared with patients with increased Wnt10a expression $(\mathrm{P}=0.019)$.

drugs, traditional surgical removal followed by chemotherapy remains the primary treatment method, despite patients 
often exhibiting recurrence following the initial therapy (2). Wnt/ $\beta$-catenin signaling is known to serve important functions in fetal development and several types of cancer (4), and $>1$ Wnt gene has been associated with the carcinogenesis of ovarian cancer (8). Wnt10a, the homolog of Wnt10b, was identified to be overexpressed in various types of tumor cells, and to function as an oncogene in renal cell carcinoma (18). However, to the best of our knowledge, the present study was the first to explore the function of Wnt10a in the carcinogenesis and progression of ovarian cancer.

According to the results of the present study, Wnt10a was highly expressed in the SKOV3 and A2780 ovarian cancer cell lines. In the loss of function assay, Wnt10a was knocked-down by a siRNA designed to target Wnt10a in SKOV3 cells. Subsequently, the viability rate and the migratory ability of SKOV 3 cells were identified to be markedly disrupted by Wnt10a siRNA. The results of the present study were consistent with the early report in renal cell carcinoma and suggested that Wnt10a may serve an oncogenic role in the carcinogenesis of ovarian cancer (18). Furthermore, in renal cell carcinoma, Wnt10a was identified to increase intracellular $\beta$-catenin accumulation through nuclear translocation, and to activate downstream molecules such as TCF4 and LEF1 (18). In the present study, the expression levels of $\beta$-catenin, cyclin D1, LEF1 and TCF4 were significantly decreased in Wnt10a-siRNA-SKOV3 cells, which was validated by LGK-974, the specific inhibitor targeting PORCN in Wnt/ $\beta$-catenin signaling (24). However, the effect of Wnt10a was independent of LGK-974 as no synergic effects between Wnt10a siRNA and LGK-974 were revealed. Therefore, the canonical Wnt/ $\beta$-catenin signaling pathway was determined to be activated by Wnt10a in ovarian cancer.

Tumorigenesis is a complex multistep process; uncontrolled cell viability and migration are two of the ten hallmarks of cancer (25). Typically, cancer cells with potent migratory ability exhibit an increased likelihood of invading adjacent healthy tissues, forming novel lesions or leading to metastasis (26). The clinical significance of Wnt10a has been revealed in renal cell carcinoma, where it was evaluated as an independent risk factor and identified to increase the incidence risk of renal carcinogenesis by 2-4-fold (18). Wnt10a is an independent risk factor for an increased mortality rate in renal cell carcinoma (18). Additionally, the Wnt10a rs7349332 polymorphism has been associated with an increased risk of developing colorectal cancer via increasing fat intake (27). In the present study, a retrospective analysis of 86 patients using RT-qPCR revealed that Wnt10a was expressed at increased levels in the tumoral tissues, as compared with in the paratumoral tissues. In addition, the overexpression of Wnt10a was identified to be significantly associated with certain clinical characteristics of ovarian cancer, including clinical tumor stage (grade III) and tumor-node-metastasis classification (T3) (20). Based on Wnt10a expression, a Kaplan-Meier estimator survival curve was constructed, which demonstrated that patients with decreased Wnt10a expression levels exhibited improved overall survival compared with patients with increased levels of Wnt10a, suggesting that Wnt10a may be a predictor for the survival of patients with ovarian cancer. These preliminary results indicated that decreased Wnt10a expression may be associated with improved prognosis in patients with ovarian cancer. However, additional studies involving a larger cohort of patients with ovarian cancer are required to validate these results.

Wnt10a-Wnt6 are clustered on the human chromosome 2 q35 region in a head-to-tail manner and were identified to be co-expressed in SW480 cells (17). However, no significant difference was identified between Wnt6 mRNA expression and Wnt10a in SKOV3 ovarian cells. Wnt10a was identified to function in an autocrine manner in renal cell carcinoma (18), and so we hypothesize that Wnt10a may also activate the $\mathrm{Wnt} / \beta$-catenin signaling pathway in an autocrine manner in ovarian cancer cells.

The results of the present study indicated that the overexpression of Wnt10a serves oncogenic functions in the promotion of carcinogenesis and progression of human ovarian cancer by activating the $\mathrm{Wnt} / \beta$-catenin/TCF/LEF1 signaling pathway. In addition, the overexpression of Wnt10a was clinically associated with high tumor grade and with late stage in patients with ovarian cancer. Wnt10a may be a predictor for the survival of patients with ovarian cancer, and serve as a novel target for drug development and as a prognostic marker.

\section{References}

1. Siegel RL, Miller KD and Jemal A: Cancer Statistics, 2015. CA Cancer J Clin 65: 5-29, 2015.

2. Bixel K and Hays JL: Olaparib in the management of ovarian cancer. Pharmacogenomics Pers Med 8: 127-135, 2015.

3. Luvero D, Milani A and Ledermann JA: Treatment options in recurrent ovarian cancer: Latest evidence and clinical potential. Ther Adv Med Oncol 6: 229-239, 2014.

4. Narita T, Sasaoka S, Udagawa K, Ohyama T, Wada N, Nishimatsu S, Takada S and Nohno T: Wnt10a is involved in AER formation during chick limb development. Dev Dyn 233: 282-287, 2005.

5. Sherwood V: WNT signaling: An emerging mediator of cancer cell metabolism? Mol Cell Biol 35: 2-10, 2015.

6. Lu D, Zhao Y, Tawatao R, Cottam HB, Sen M, Leoni LM, Kipps TJ, Corr M and Carson DA: Activation of the Wnt signaling pathway in chronic lymphocytic leukemia. Proc Natl Acad Sci USA 101: 3118-3123, 2004.

7. Hilliard TS, Gaisina IN, Muehlbauer AG, Gaisin AM, Gallier F and Burdette JE: Glycogen synthase kinase $3 \beta$ inhibitors induce apoptosis in ovarian cancer cells and inhibit in vivo tumor growth. Anticancer Drugs 22: 978-985, 2011.

8. Arend RC,Londoño-Joshi AI, Straughn JM Jr and Buchsbaum DJ: The Wnt/ $\beta$-catenin pathway in ovarian cancer: A review. Gynecol Oncol 131: 772-779, 2013.

9. Gatcliffe TA, Monk BJ, Planutis K and Holcombe RF: Wnt signaling in ovarian tumorigenesis. Int J Gynecol Cancer 18: 954-962, 2008.

10. Wu R, Zhai Y, Fearon ER and Cho KR: Diverse mechanisms of beta-catenin deregulation in ovarian endometrioid adenocarcinomas. Cancer Res 61: 8247-8255, 2001.

11. Burkhalter RJ, Symowicz J, Hudson LG, Gottardi CJ and Stack MS: Integrin regulation of beta-catenin signaling in ovarian carcinoma. J Biol Chem 286: 23467-23475, 2011.

12. Yoshioka S, King ML, Ran S, Okuda H, MacLean JA II, McAsey ME, Sugino N, Brard L, Watabe K and Hayashi K: WNT7A regulates tumor growth and progression in ovarian cancer through the WNT//-catenin pathway. Mol Cancer Res 10: 469-482, 2012

13. Chen H, Wang Y and Xue F: Expression and the clinical significance of Wnt10a and Wnt10b in endometrial cancer are associated with the Wnt/ $\beta$-catenin pathway. Oncol Rep 29: 507-514, 2013.

14. Bonds J, Pollan-White S, Xiang L, Mues G and D'Souza R: Is there a link between ovarian cancer and tooth agenesis? Eur J Med Genet 57: 235-239, 2014.

15. Kirikoshi $\mathrm{H}$, Inoue $\mathrm{S}$, Sekihara $\mathrm{H}$ and Katoh M: Expression of WNT10A in human cancer. Int J Oncol 19: 997-1001, 2001.

16. Long A, Giroux V, Whelan KA, Hamilton KE, Tétreault MP, Tanaka K, Lee JS, Klein-Szanto AJ, Nakagawa H and Rustgi AK: WNT10A promotes an invasive and self-renewing phenotype in esophageal squamous cell carcinoma. Carcinogenesis 36: 598-606, 2015. 
17. Kirikoshi H, Sekihara H and Katoh M: WNT10A and WNT6, clustered in human chromosome 2 q35 region with head-to-tail manner, are strongly coexpressed in SW480 cells. Biochem Biophys Res Commun 283: 798-805, 2001.

18. Hsu RJ, Ho JY, Cha TL, Yu DS, Wu CL, Huang WP, Chu P, Chen YH, Chen JT and Yu CP: WNT10A plays an oncogenic role in renal cell carcinoma by activating WNT/ $\beta$-catenin pathway. PLoS One 7: e47649, 2012

19. Kirikoshi H, Sekihara H and Katoh M: Up-regulation of WNT10A by tumor necrosis factor $\alpha$ and Helicobacter pylori in gastric cancer. Int J Oncol 19: 533-536, 2001.

20. Scully RE: World Health Organization classification and nomenclature of ovarian cancer. Natl Cancer Inst Monogr 42: 5-7, 1975.

21. Shepherd JH: Revised FIGO staging for gynaecological cancer. Br J Obstet Gynaecol 96: 889-892, 1989.

22. Denny L, Quinn M and Hacker N: FIGO cancer report 2012. Int J Gynaecol Obstet 119 (Suppl 2): S89, 2012.

23. Livak KJ and Schmittgen TD: Analysis of relative gene expression data using real-time quantitative PCR and the 2(-Delta Delta C(T)) method. Methods 25: 402-408, 2001
24. Liu J, Pan S, Hsieh MH, Ng N, Sun F, Wang T, Kasibhatla S, Schuller AG, Li AG, Cheng D, et al: Targeting Wnt-driven cancer through the inhibition of Porcupine by LGK974. Proc Natl Acad Sci USA 110: 20224-20229, 2013.

25. Hanahan D and Weinberg RA: Hallmarks of Cancer: The next generation. Cell 144: 646-674, 2011.

26. Entschladen F, Drell TL IV, Lang K, Joseph J and Zaenker KS: Tumour-cell migration, invasion, and metastasis: Navigation by neurotransmitters. Lancet Oncol 5: 254-258, 2004.

27. Galbraith RL, Poole EM, Duggan D, Muehling J, Hsu L, Makar K, Xiao L, Porter JD and Ulrich CM: Polymorphisms in WNT6 and WNT10A and colorectal adenoma risk. Nutr Cancer 63: 558-564, 2011. 